\title{
Mechanism of Localization of the Magnetization Reversal in 3 nm Wide Co Nanowires
}

\author{
F. Vidal, ${ }^{1,2}$ Y. Zheng, ${ }^{1,2}$ P. Schio, ${ }^{1,3}$ F. J. Bonilla, ${ }^{1}$ M. Barturen,${ }^{1,2,4}$ J. Milano,,${ }^{1,2,4}$ D. Demaille, ${ }^{1}$ E. Fonda, ${ }^{5}$ \\ A. J. A. de Oliveira, ${ }^{3}$ and V. H. Etgens ${ }^{1,6}$ \\ ${ }^{1}$ Institut des NanoSciences de Paris, UMR CNRS 7588, UPMC Université Paris 6, 4 Place Jussieu 75005 Paris, France \\ ${ }^{2}$ LIFAN, Laboratoire International Franco-Argentin en Nanosciences, Argentina, France \\ ${ }^{3}$ Departamento de Física, UFSCar, C. P. 676, 13565-905 São Carlos, São Paulo, Brazil \\ ${ }^{4}$ CNEA-CONICET and Instituto Balseiro, UNCU. CAB (R8402AGP) San Carlos de Bariloche, Río Negro, Argentina \\ ${ }^{5}$ Synchrotron Soleil, L'Orme des Merisiers Saint-Aubin BP 48, 91192 Gif-sur-Yvette Cedex, France \\ ${ }^{6}$ Fédération Lavoisier Franklin, UVSQ, 45 Avenue des Etats Unis-78035 Versailles Cedex, France
}

(Received 9 February 2012; published 14 September 2012; publisher error corrected 18 September 2012)

The mechanism of magnetization reversal has been studied in a model system of self-assembled cobalt nanowires with a $3 \mathrm{~nm}$ diameter. The structure, orientation and size of grains within the nanowires could be determined by high resolution transmission electron microscopy. The magnetic properties were probed using static and dynamic magnetization measurements. Micromagnetic modeling based on the structural analysis allows us to correlate the structure and the magnetic behavior of the wires, revealing competition between shape anisotropy, magnetocrystalline anisotropy and exchange in the localized reversal within Co hcp oriented grains. These results provide direct experimental evidence of the link between anisotropy fluctuations and reversal localization in nanowires.

DOI: 10.1103/PhysRevLett.109.117205

PACS numbers: 75.30.Gw, 75.75.-c, 81.07.Gf

Ferromagnetic nanowires have drawn considerable interest in the past few years because of their potential applications in data storage devices [1-6]. The magnetic anisotropy is dominated by shape effects in a nanowire, leading to two stable configurations of its overall magnetic moment aligned along the axis of the wire. These two preferential orientations make it an ideal fundamental unit to store a bit in a data storage system. Due to the reduced diameter of nanowires, it is foreseen that their use may lead to increased storage densities.

However, there are hurdles to be overcome before such objects can find their place in our everyday life. One important step is to fully understand the relationship between the structure at the nanoscale and the magnetic properties. In this respect, nanowires-with a diameter falling below characteristic magnetic length scales such as the exchange length or the domain wall widthrepresent a testing ground in the field of nanomagnetism. Theoretically, nanowires with very small diameters are expected to behave as macrospins, keeping all their magnetic moments aligned and flipping as a single moment when the field is reversed. Experimentally, this is not observed and activation energies for reversal extracted from magnetic measurements point towards a localization of the reversal [2]: the magnetization starts to reverse in a limited portion of the wire as opposed to a reversal at unison in the whole wire. Understanding the details of this localization phenomenon is a key point both at a fundamental level and for applications.

The real structure of ferromagnetic nanowires (FNWs), accompanied by deviation from ideality (such as crystalline imperfection, diameter variation or misorientation), is thought to be responsible for the localization of the reversal through local variation of the anisotropy [7], as illustrated in Fig. 1(a). However, direct evidence of this link is still missing and attempts to correlate the real inner structure and properties at the nanoscale, an ongoing challenge of materials science, in ferromagnetic nanowires are scarce [8-13]. In this Letter, we study a model system made of (a)
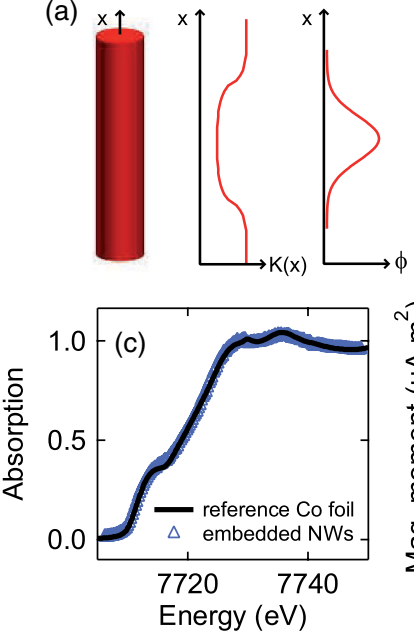

(b)

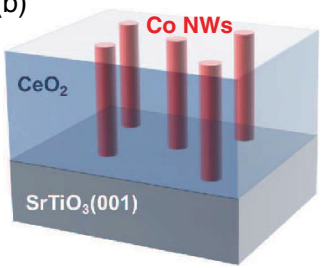

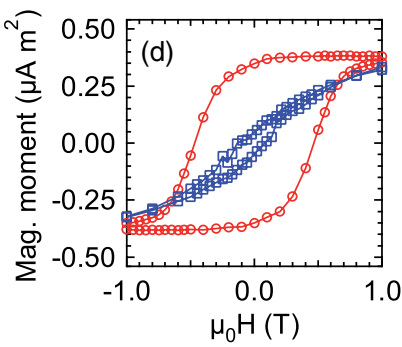

FIG. 1 (color online). (a) Localization of the magnetization reversal due to anisotropy $(K)$ fluctuations along the wire axis, $\phi$ is the angle of the local magnetization with respect to the FNW axis during reversal. (b) Schematic of the FNW assembly studied in this Letter. (c) X-ray absorption spectra at the Co $K$ edge of the FNW assembly and of a reference Co hcp foil. (d) Magnetic hysteresis cycles at $20 \mathrm{~K}$ with field parallel (circles) and perpendicular (squares) to the wires. 
ultranarrow cobalt wires ( $3 \mathrm{~nm}$ diameter) embedded in a $\mathrm{CeO}_{2} / \mathrm{SrTiO}_{3}(001)$ epilayer, obtained by vertical selfassembly. We evidence the localization of the magnetization reversal induced by fluctuation of the local anisotropy in this model system and correlate intimately the internal structure of the wires with the reversal mechanism.

The self-assembled embedded Co FNWs studied here, represented schematically in Fig. 1(b), form spontaneously during pulsed-laser deposition of Co-doped ceria $\left(\mathrm{CeO}_{2}\right)$ epitaxial films on $\mathrm{SrTiO}_{3}(001)$. Details on the elaboration and basic magnetization measurements can be found elsewhere $[14,15]$. With respect to our previous works evidencing the formation of nanowires and a possible magnetocrystalline anisotropy contribution to the wires anisotropy, the results presented in this Letter allow us to gain physical insight into the reversal process through major advances in the structure determination, magnetic anisotropy study, and micromagnetic modeling. The nanowire assembly studied here has a narrow diameter distribution with a mean diameter of $3 \mathrm{~nm}$ and the distribution of misorientations with respect to the growth direction, [001], is also narrow [15]. The metallic character of the nanowires is attested by the X-ray absorption spectrum taken at Co $K$ edge in Fig. 1(c). The easy magnetic axis of the FNWs assembly lies along the [001] direction, as shown by hysteresis cycles in Fig. 1(d). The structure of the FNWs was studied using high resolution transmission electron microscopy (HRTEM). Magnetic measurements were performed in a superconducting quantum interference device magnetometer (SQUID, MPMS 5S Quantum Design) and ferromagnetic resonance was performed in the $Q$ band $(35 \mathrm{GHz})$ using a Bruker spectrometer.

Figure 2(a) shows a representative HRTEM image taken along the ceria $[1 \overline{1} 0]$ zone axis, where a perturbed area oriented along [001], due to the presence of a FNW, can be observed. Performing fast Fourier transform (FFT) locally along the FNW reveals the distinct patterns depicted in Figs. 2(b)-2(d). Such patterns were consistently observed in HRTEM images recorded in different area and pieces of the sample. The pattern in Fig. 2(c) is the least frequently observed, corresponding to a minority structure. It can be indexed, revealing a cube-on-cube epitaxy of fcc Co with ceria. The spots of the matrix (blue circles) are aligned with the spots of a cubic structure with a $3.54 \AA$ lattice parameter (red squares). The remaining spots are obtained by considering multiple diffraction (each diffracted beam in the matrix can be diffracted as a primary beam in the embedded object). Thus, locally in some minor portions of the FNWs, Co is present in its cubic form, aligned with $\mathrm{CeO}_{2}$.

The other patterns can then be indexed considering the fcc-hcp transition of Co. During this transition, the [111] fcc direction is transformed into the [0001] direction of the hcp structure ( $c$ axis). Labeling $\mathbf{C}^{\star}$ as the base vector along the hexagonal axis of the reciprocal lattice of hcp Co, the
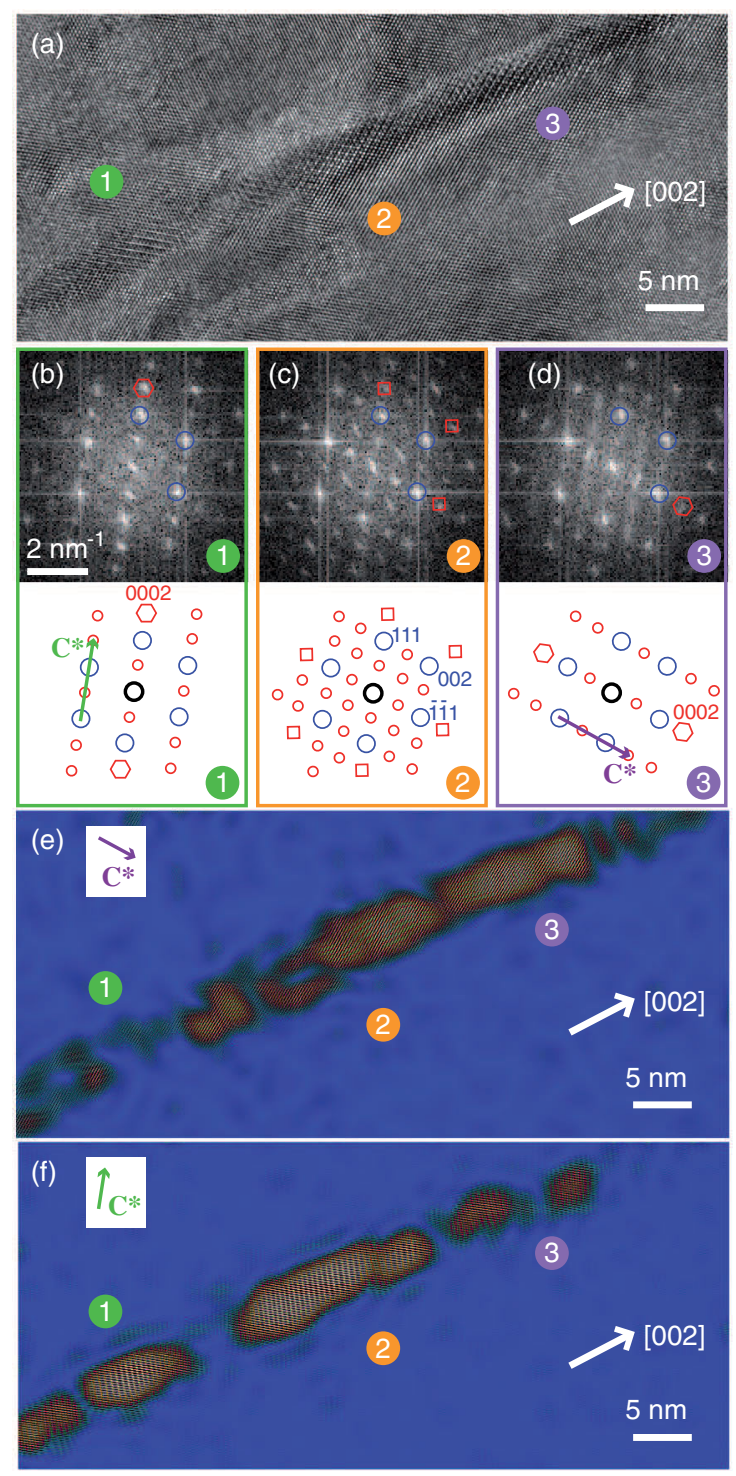

FIG. 2 (color online). (a) HRTEM cross-sectional image of an embedded FNW taken along the ceria [1ㅣㅣ zone axis. (b)-(d) Local diffraction patterns obtained by FFT in zones labeled 1-3 in (a), with the indexation of these patterns considering multiple diffraction in ceria and hcp Co with $c$ axis \| [111] (b), fcc Co aligned with ceria (c), hcp Co with $c$ axis \| [ $\left.\begin{array}{lll}1 & 1 & 1\end{array}\right]$ (d). (e),(f) Filtered images (on 0002 Co hcp spots) revealing the distribution of tilted hcp grains inside the FNW.

patterns of Figs. 2(b) and 2(d) can be indexed by considering hop Co with $\mathbf{C}^{\star} \|$ [111] and $\mathbf{C}^{\star} \|$ [1 111$]$, respectively. Filtering the HRTEM images on the hcp 0002 spots allows us to reveal the spatial extension of the hep Co grains along the FNW axis, as shown in Figs. 2(e) and 2(f). This structural analysis shows that the FNWs are made of Co hcp grains with their hexagonal $c$ axis aligned along a $\langle 111\rangle$ direction of the matrix (within $\pm 3^{\circ}$ from repeated measurements performed on distinct nanowires). These grains are separated by fcc regions or stacking faults and multiple twinning. The uniaxial magnetocrystalline 
anisotropy (MCA) of hcp Co, $K_{1 u}$, is of the same order of magnitude than the shape anisotropy of the FNWs, $K_{s}$. By contrast, the MCA of fcc Co is much weaker. Therefore, in tilted hcp grains ( $c$ axis at $54.7^{\circ}$ from the FNW axis), $K_{1 u}$ and $K_{s}$ will compete. Thus, the internal structure of the wires results in a system with strong fluctuations of the local anisotropy along the axis. We now turn to the study of the magnetization reversal.

The evolution of the coercive field, measured along the easy axis, with the temperature is depicted in Fig. 3(a). The data could not be fitted properly using Sharrock's formula,
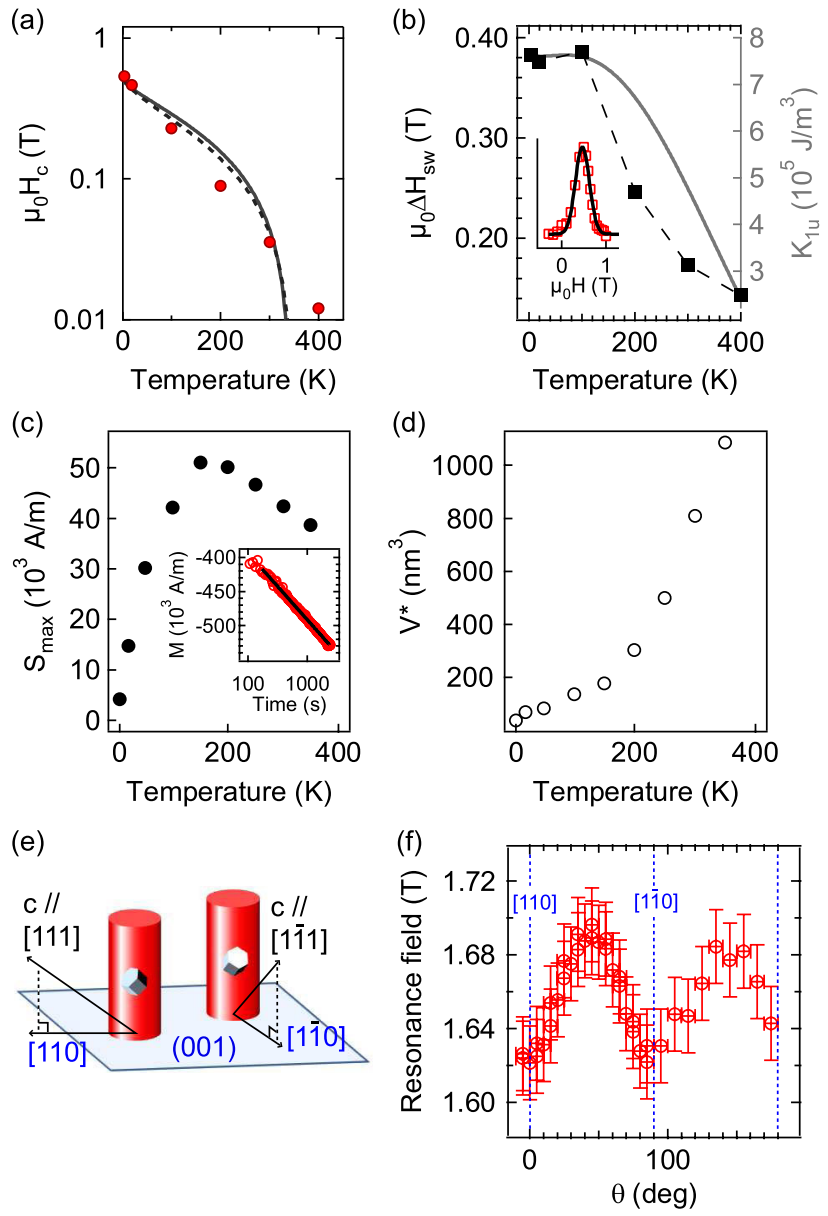

FIG. 3 (color online). (a) Variation of the coercive field with the temperature (disks) and fits assuming a temperature independent energy barrier for reversal (black line $m=3 / 2$ and dashed line $m=2$ ). (b) Width of the switching field distribution $\mu_{0} \Delta H_{\mathrm{sw}}$ (squares) and magnetocrystalline anisotropy $\left(K_{1 u}\right)$ of hcp Co (thick gray line) as a function of the temperature. Inset: switching field distribution at $T=20 \mathrm{~K}$. (c) Magnetic viscosity as a function of the temperature. Inset: magnetization decay obtained at $300 \mathrm{~K}$ and $0.0625 \mathrm{~T}$. (d) Variation of the activation volume for reversal as a function of the temperature. (e) Schematic illustrating the directions of the $c$ axis in two hcp grains within FNWs and their projection in the (001) plane. (f) Angular dependence of the resonance field recorded by FMR in the (001) plane. $\mu_{0} H_{c}=\mu_{0} H_{0}\left[1-\left(25 k_{B} T / E\right)^{1 / m}\right][16]$ and considering a temperature independent energy barrier $E$ for reversal, neither with $m=2$ nor with $m=3 / 2$. This indicates that the energy barrier depends on the temperature. As the magnetostatic contributions to the anisotropy scale as $M_{s}^{2}$ ( $M_{s}$ saturation magnetization) which is constant in the temperature range studied, this variation of $E$ with $T$ can be related to the $K_{1 u}(T)$ variation, reproduced in Fig. 3(b) along with the width of the switching field distribution, $\mu_{0} \Delta H_{\mathrm{sw}}(T) . \mu_{0} \Delta H_{\mathrm{sw}}(T)$ is constant at low temperature and drops beyond $150 \mathrm{~K}$. This temperature corresponds also to the onset of a drop in $K_{1 u}(T)$. The switching field distribution is closely related to inhomogeneities in the system. In the present case, the wires are made of tilted hcp grains where $K_{1 u}$ and $K_{s}$ compete locally, giving rise to a distribution of energy barriers. According to this picture, the weakening of $K_{1 u}(T)$ induces a drop of $\mu_{0} \Delta H_{\mathrm{sw}}(T)$.

In order to get more insight into the reversal mechanism, magnetic viscosity measurements were carried out [17]. The magnetic viscosity $S(H, T)$ was extracted from magnetization decay $\left[M(t)=M\left(t_{0}\right)-S(H, T) \ln \left(t / t_{0}\right)\right]$ performed in a broad range of temperatures and fields. $S_{\max }(T)$, the maximal value of the magnetic viscosity at a given temperature $T$, is plotted in Fig. 3(c). In the case of a temperature independent distribution of energy barriers, $S_{\max }(T)$ should depend linearly on $T$ [17]. Clearly, this is not the case and a drop in $S_{\max }(T)$ is observed beyond $150 \mathrm{~K}$. More information on the reversal can be extracted from $S$ and from the irreversible part of the susceptibility $\chi_{\text {irr }}$ [18]. The activation volume for reversal $V^{\star}$ can be derived using the relation $V^{\star}=k_{B} T \chi_{\text {irr }} / \mu_{0} M_{s} S$. The results are given in Fig. 3(d). $V^{\star}$ is much smaller than the physical volume of a FNW $\left(\sim 2500 \mathrm{~nm}^{3}\right)$, indicating that the reversal is localized. Again, two regimes are observed, below and above $150 \mathrm{~K}$ [19]. The weakening of the localization of the reversal at higher $T$ can be related to the drop of $K_{1 u}$ that leads to a damping of local variations of the anisotropy along the wires axis.

The fact that $K_{1 u}$ contributes to the anisotropy of the system has been confirmed by ferromagnetic resonance measurements. If the magnetic anisotropy contains only dipolar contributions, then the resonance field probed in the plane of the epilayer (perpendicular to the wires) should not depend on the direction of the applied magnetic field. By contrast, a $K_{1 u}$ contribution with the $c$ axis parallel to the $\langle 111\rangle$ directions of the matrix should lead to the appearance of in plane easy axis (with lower resonance fields) along the [110] and [1ํㅣㄹ directions, as illustrated in Fig. 3(e). This is exactly what is observed, as shown in Fig. 3(f), confirming the MCA contribution to the total magnetic anisotropy.

Using inputs from the structural and magnetic studies, we now turn to the modeling of the localized reversal of magnetization and determination of the nucleation field in the FNWs. In grains having their $c$ axis tilted with respect 
to the FNWs axis, the MCA of hcp Co could play against the shape anisotropy. We thus make the assumption that the localized reversal may occur within such tilted grains. The magnetic anisotropies are described as shown in Fig. 4(a): we treat the FNWs in the framework of a mean field approximation, gathering the shape anisotropy and dipolar interactions in a single magnetostatic anisotropy, $K_{m}$, and we consider a distribution of MCA reaching its maximum value $\left(K_{1 u}\right)$ at the middle of an oriented grain and vanishing outside. The shape anisotropy is given by $K_{S}=$ $\left(\mu_{0} / 4\right) M_{s}^{2}$. The other magnetostatic contribution, $K_{\text {dip }}$, is related to the dipolar interactions between wires that also scales as $M_{s}^{2}$. It has been shown that $K_{S}$ and $K_{\text {dip }}$ can be merged in a single contribution as $K_{m}=K_{S}+K_{\text {dip }}=$ $\left(\mu_{0} / 4\right) M_{s}^{2}(1-3 P)$ where $P$ is the porosity (volume fraction occupied by the wires) of the FNWs assembly [20].

The magnetic free energy of a FNW, $E$, can then be expressed as:

$$
\frac{E}{s}=\int\left\{A\left(\frac{\partial \phi}{\partial x}\right)^{2}+E_{a}(\phi, x)-\mu_{0} M_{s} H \cos \phi\right\} d x
$$

where $s=\pi R^{2}$ is the section of FNW with radius $R, A$ is the exchange stiffness, and $\phi$ is the angle between the magnetic moments and $H$ applied along the wires axis. $E_{a}$ is the anisotropy energy density, modeled as:

$$
E_{a}(\phi, x)=-K_{m} \cos ^{2} \phi-K_{u}(x) \cos ^{2}(\phi-\theta)
$$

with $K_{u}(x)$ the position-dependent contribution of Co MCA and $\theta=54.7^{\circ}$ the angle between the $c$ axis and the wire axis, according to our structural study. Taking $K_{u}(x)=K_{1 u} \exp \left(-x^{2} / \ell^{2}\right)$ allows us to model the contribution of a hcp grain inserted between two regions where the effect of magnetocrystalline anisotropy vanishes (grain boundaries, stacking faults, fcc grains...). Minimization of this magnetic free energy can be achieved through solving the corresponding Euler equation [21,22]. The nucleation field $\mu_{0} H_{n}$ can then be determined as:

$$
\mu_{0} H_{n}=\frac{2 K_{m}}{M_{s}}-\frac{2 K_{1 u}|\cos 2 \theta|}{M_{s}}+\frac{\delta_{\mathrm{DW}}}{\pi \ell} \frac{2 K_{1 u} \sqrt{|\cos 2 \theta|}}{M_{s}}
$$

where $\delta_{\mathrm{DW}}=\pi \sqrt{A / K_{1 u}}$ is the domain wall width in hep Co. $\mu_{0} H_{n}$ can be written as the sum of three terms, each of these three terms can be identified: the first one is related to shape anisotropy, the second is a drop related to the effect of magnetocrystalline anisotropy in the grain with tilted $c$ axis that plays against shape anisotropy, the last one is related to the increase in exchange energy at the borders of the flipping region where magnetic moments are not parallel anymore.

In order to evaluate $K_{m}$, we need to take into account the dipolar coupling between wires. Following Ref. [20], from the measured value of the porosity $P$ of the assembly, we get $K_{m}=5.51 \times 10^{5} \mathrm{~J} \cdot \mathrm{m}^{-3}$. We also performed mean field calculations of the dipolar field, considering an assembly of $150 \mathrm{~nm}$-long FNWs randomly distributed where the density was set to the one measured by TEM [from analysis of plane view images such as the one in Fig. 4(b)]. The area of the considered assembly was $7 \times 7 \mu \mathrm{m}^{2}$. The wire length was set equal to half of the film thickness. We also estimated the influence of the FNWs' length by performing additional calculations for $300 \mathrm{~nm}$-long and $75 \mathrm{~nm}$-long FNWs and found that the dipolar magnetic energy changes only by about $5 \%$. On the other hand, the energy is highly dependent on the nanowire density (related to the mean distance between wires) and on the FNW magnetization. From such calculations, we get a mean value of $K_{m}=5.45 \times 10^{5} \mathrm{~J} \cdot \mathrm{m}^{-3}$, in very good agreement with the preceding result. Although the method used to evaluate the dipolar coupling may be crude, the estimated value of the dipolar field, $\mu_{0} H_{\text {dip }}=2 K_{\text {dip }} / M_{s}=$ $0.14 \mathrm{~T}$, is consistent with the width of switching field distribution at $300 \mathrm{~K}(0.17 \mathrm{~T})$ which gives an upper bound for $\mu_{0} H_{\text {dip }}$ [as $\mu_{0} \Delta H_{\text {sw }}(T)$ results from dipolar coupling and an intrinsic width related to the wires structure].

Using the values available in the literature for the magnetic parameters of $\mathrm{Co}$, the value of $\mu_{0} H_{n}$ can then be evaluated. For $\ell=10 \mathrm{~nm}$, we get $\mu_{0} H_{n}=0.63 \mathrm{~T}$ which matches very well with the coercive field extrapolated at $0 \mathrm{~K}(\sim 0.6 \mathrm{~T})$. Also, the value of $\ell$ is consistent with the size of the hcp grains detected in TEM images which is of the order of 5-20 nm, as shown in Fig. 4(c). Moreover, grains of $10 \mathrm{~nm}$ length and $3 \mathrm{~nm}$ diameter correspond to volumes of $\sim 70 \mathrm{~nm}^{3}$. This is fully consistent with the activation volume at low temperature extracted from viscosity measurements, Fig. 3(d).

From the model, we can deduce also the effective magnetocrystalline anisotropy inside the wires, given by:

$$
K_{\mathrm{eff}}=K_{1 u}\left(1-\frac{\delta_{\mathrm{DW}}}{\pi \ell \sqrt{|\cos 2 \theta|}}\right)
$$

The magnitude $K_{\text {eff }}$ is lower than $K_{1 u}$ due to the extra stiffness added by exchange at the border of the grains. This trend is confirmed by preliminary analysis of the FMR measurements that leads to a magnetocrystalline anisotropy contribution lower than $K_{1 u}$.
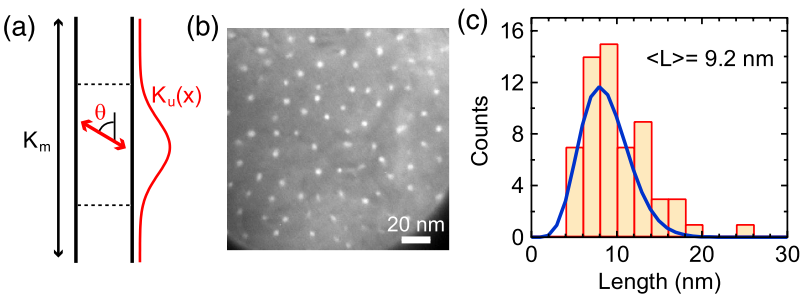

FIG. 4 (color online). (a) Model anisotropy distribution used to compute the nucleation field. (b) Typical energy filtered plane view TEM image used to measure the mean distance between wires. (c) Size distribution of the hcp Co grains. 
Before concluding, we comment on the origin of the drop in $\mu_{0} H_{c}(T)$ observed at higher temperature. Because of the localization of the reversal, the nanowires can be viewed as entities with effective anisotropy and volume, leading to a finite blocking temperature. Above this blocking temperature, higher than $400 \mathrm{~K}$ here, the coercive field vanishes and the system is superparamagnetic. However, this point may deserve further investigation as other effects could be the source of the high temperature behavior. Considering the reduced diameter of the nanowires, finite size effects may also play a role here $[23,24]$. Indeed, in $\mathrm{Ni}$ FNWs, a dramatic decrease of the Curie temperature when the diameter is reduced has already been reported [25]. This effect arises because the diameter of the FNWs acts as a cutoff for the correlation length at the ferromagnetic transition. In the case of $3 \mathrm{~nm}$ diameter cobalt nanowires, estimations of the Curie temperature, using data available from size effects studies on ultrathin Co films [24,26,27], indicate that the Curie temperature should remain substantially higher than $400 \mathrm{~K}$.

In conclusion, the mechanism of localization of the magnetization reversal could be determined and correlated to the real nanoscale wire structure of a Co FNW assembly, establishing the link between anisotropy fluctuation and reversal localization. From these results, it is anticipated that the control of the wire structure (increase of grain size or stabilization of the fcc phase) could lead to a behavior closer to reversal at unison. Further studies are currently in progress in order to control the structure of self-assembled embedded wires, as well as to explore its impact on the magnetic properties.

We thank M. Audier, LMGP, CNRS, Grenoble, for illuminating discussions, F. Breton for skillful help and design of the PLD control system. This work has been supported by the Region Ile-de-France in the framework of C'Nano IdF. C'Nano IdF is the nanoscience competence center of Paris Region, supported by CNRS, CEA, MESR and Region Ile-de-France. We also acknowledge support from ANR, Contract No. ANR-2011-BS04-007-01. We thank B. Capelle and J.-M. Guigner, IMPMC, CNRSUPMC, for access to the TEM facilities. P.S. acknowledges support from FAPESP (Grant No. 2007/08649-5).

[1] A. Fert and L. Piraux, J. Magn. Magn. Mater. 200, 338 (1999).

[2] D. J. Sellmyer, M. Zheng, and R. Skomski, J. Phys. Condens. Matter 13, R433 (2001).

[3] L. Sun, Y. Hao, C.-L. Chien, and P. C. Searson, IBM J. Res. Dev. 49, 79 (2005).
[4] J.-H. Gao, D.-L. Sun, Q.-F. Zhan, W. He, and Z.-H. Cheng, Phys. Rev. B 75, 064421 (2007).

[5] T. Maurer, F. Zighem, F. Ott, G. Chaboussant, G. André, Y. Soumare, J.-Y. Piquemal, G. Viau, and C. Gatel, Phys. Rev. B 80, 064427 (2009).

[6] X. Kou, X. Fan, R. K. Dumas, Q. Lu, Y. Zhang, H. Zhu, X. Zhang, K. Liu, and J. Q. Xiao, Adv. Mater. 23, 1393 (2011).

[7] R. Skomski, H. Zeng, M Zheng, and D. J. Sellmyer, Phys. Rev. B 62, 3900 (2000).

[8] M. Zheng, R. Skomski, Y. Liu, and D. J. Sellmyer, J. Phys. Condens. Matter 12, L497 (2000).

[9] H. Zeng, R. Skomski, L. Menon, Y. Liu, S. Bandyopadhyay, and D. J. Sellmyer, Phys. Rev. B 65, 134426 (2002).

[10] Z. Liu, P.-C. Chang, C.-C. Chang, E. Galaktionov, G. Bergmann, and J.G. Lu, Adv. Funct. Mater. 18, 1573 (2008).

[11] M. Darques, L. Piraux, A. Encinas, P. Bayle-Guillemaud, A. Popa, and U. Ebels, Appl. Phys. Lett. 86, 072508 (2005).

[12] K. Soulantica, F. Wetz, J. Maynadié, A. Falqui, R. P. Tan, T. Blon, B. Chaudret, and M. Respaud, Appl. Phys. Lett. 95, 152504 (2009).

[13] K. R. Pirota, F. Béron, D. Zanchet, T. C. R. Rocha, and D. Navas, J. Torrejón, M. Vazquez, and M. Knobel, J. Appl. Phys. 109, 083919 (2011).

[14] F. Vidal, Y. Zheng, J. Milano, D. Demaille, P. Schio, E. Fonda, and B. Vodungbo, Appl. Phys. Lett. 95, 152510 (2009).

[15] P. Schio, F. Vidal, Y. Zheng, J. Milano, E. Fonda, D. Demaille, B. Vodungbo, J. Varalda, A. J. A. de Oliveira, and V.H. Etgens, Phys. Rev. B 82, 094436 (2010).

[16] M. P. Sharrock, J. Appl. Phys. 76, 6413 (1994).

[17] R. Skomski, J. Phys. Condens. Matter 15, R841 (2003).

[18] P. Gaunt, J. Appl. Phys. 59, 4129 (1986).

[19] The variation of $K_{1 u}$ with temperature is mainly related to the thermal evolution of the $c / a$ ratio in hcp Co, see F. Ono and H. Maeta, Physica B (Amsterdam) 161, 134 (1989).

[20] A. Encinas-Oropesa, M. Demand, L. Piraux, I. Huynen, and U. Ebels, Phys. Rev. B 63, 104415 (2001).

[21] P. Bruno, Phys. Rev. Lett. 83, 2425 (1999).

[22] H. Kronmüller, Phys. Status Solidi B 144, 385 (1987).

[23] C. M. Schneider, P. Bressler, P. Schuster, J. Kirschner, J. J. de Miguel, and R. Miranda, Phys. Rev. Lett. 64, 1059 (1990).

[24] C. A. F. Vaz, J. A. C. Bland, and G. Lauhoff, Rep. Prog. Phys. 71, 056501 (2008).

[25] L. Sun, P. C. Searson, and C. L. Chien, Phys. Rev. B 61, R6463 (2000).

[26] F. Huang, G. J. Mankey, M. T. Kief, and R. F. Willis, J. Appl. Phys. 73, 6760 (1993).

[27] F. Huang, M. T. Kief, G. J. Mankey, and R. F. Willis, Phys. Rev. B 49, 3962 (1994). 